\title{
A TECNOLOGIA ASSISTIVA COMO FACILITADORA NO PROCESSO DE INCLUSÃO: DAS POLÍTICAS PÚBLICAS A LITERATURA
}

\author{
LA TECNOLOGÍA ASISTIVA COMO FACILITADORA EN EL PROCESO DE \\ INCLUSIÓN: DE LAS POLÍTICAS PÚBLICAS A LA LITERATURA
}

\section{ASSISTIVE TECHNOLOGY AS SUPPORT FOR THE INCLUSION PROCESS: FROM PUBLIC POLICIES TO LITERATURE}

RESUMO: A política educacional brasileira garante a matrícula dos alunos público alvo da educação especial em escolas comuns, porém a inclusão escolar de parte desse público tem apresentado um desafio para os profissionais na escola, a utilização da Tecnologia Assistiva é vista como facilitador no processo de inclusão. Dessa forma esse estudo pretende por meio de uma revisão bibliográfica apontar a importância da Tecnologia Assitiva no processo de inclusão. Os resultados mostram a necessidade de formação profissional, bem como, políticas de financiamento e implementação efetiva desse importante recurso para inclusão de todos.

PALAVRAS-CHAVE: Tecnologia assistiva. Inclusão. Alunos público-alvo da educação especial.

RESUMEN: La política educativa brasileña garantiza la matrícula de los alumnos público blanco de la educación especial en escuelas comunes, pero la inclusión escolar de parte de ese público ha presentado un desafio para los profesionales en la escuela, la utilización de la Tecnologia Asistiva es vista como facilitadora en el proceso de inclusión. De esa forma ese estudio pretende por medio de un recogido bibliográfico apuntar la importancia de la Tecnología Asistiva en el proceso de inclusión. Los resultados muestran la necesidad de formación profesional, bien como, políticas de financiamiento e implementación efetiva de ese importante recurso para inclusión de todos.

PALAVRAS-CLAVE: Tecnología asistiva. Inclusión. Alumnos público blanco da educación especial.

ABSTRACT: The Brazilian educational policy ensures the registration of student gives special education in schools, but of these students presents a challenge for professionals at school,use Assistive Technology make it easy the process of inclusion. In this way, this

${ }^{1}$ Universidade Estadual Paulista (Unesp), Araraquara - SP - Brasil. Mestranda em Educação Escolar. Email: tamirisfachinetti@gmail.com.

${ }^{2}$ Universidade Estadual Paulista (Unesp), Araraquara - SP - Brasil. Docente do Departamento de Psicologia da Educação. Doutora em Educação Especial. E-mail: relmaurel@fclar.unesp.br.

RPGE- Revista on line de Política e Gestão Educacional, Araraquara, v.21, n.esp.3, p. 1588-1597, dez., 2017. 
study to intend with one literature review show the importance of Assistive Technology in the process of inclusion. The results show the need for vocational training, financing policies, and effective implementation of this important resource for the inclusion of all.

KEYWORDS: Assistive technology. Inclusion. Student gives special education.

\section{Introdução}

A literatura tem mostrado uma demanda crescente sobre referenciais teóricos que abordem a Tecnologia Assistiva (TA), no entanto percebe-se que ainda não à uma consistência conceitual sobre o tema no Brasil, além de não haver um uso efetivo da TA no ambiente educacional. Varela e Oliver (2013) alertam para a necessidade do desenvolvimento de pesquisas que justifiquem a importância da TA e caracterize o uso da mesma na vida cotidiana das pessoas com deficiência. A TA é um elemento chave para promoção dos direitos das pessoas com deficiência.

Subsidiadas por essas referências e com o intuito de expandir a discussão acerca da TA como facilitadora no processo educacional inclusivo esse estudo têm como objetivo abordar considerações sobre a TA no cenário educacional brasileiro, tendo como base para essa discussão as principais políticas públicas que garantem esse serviço bem como estudos que legitimam a importância da interface de seu uso na educação inclusiva.

Para que esse estudo se concretizasse optou-se em utilizar uma investigação de abordagem qualitativa em relação ao seu modo de abordagem, de caráter exploratório descritivo no que se refere ao seu objetivo geral e de natureza bibliográfica por conta dos procedimentos técnicos adotados em sua realização. Um estudo exploratório pode ser entendido como um ponto de partida para futuras pesquisas já que leva ao conhecimento aprofundado de determinado fenômeno delimitando fatos e oferecendo subsídios de embasamento sobre o assunto do estudo.

\section{Tecnologia assistiva}

Segundo Rodrigues e Alves (2013) durante muito tempo a discussão sobre TA ficou restrita a profissionais da área da saúde devido ao viés de reabilitação presente na questão da funcionalidade que é vital para a utilização da TA. No ambiente educacional alguns pesquisadores utilizavam termos como mobiliários ou recursos pedagógicos 
adaptados, recursos de comunicação alternativa, acessibilidade e informática acessível, entre outros, para se referir ao que hoje, depois de diferentes terminologias utilizadas, chamamos de TA (MANZINI, 2011a).

No Brasil é possível ver um maior interesse e investimento científico na área da TA a partir de 1990 (CASTRO; SOUZA; SANTOS, 2011), o termo ajudas técnicas foi utilizado como sinônimo da TA por algum tempo. O Artigo 61 do Decreto Lei no 5296 de 02/12/2004, que regulamentou a Política Nacional para a Integração da pessoa com deficiência, definiu ajudas técnicas como: "produtos, instrumentos, equipamentos ou tecnologia adaptados ou especialmente projetados para melhorar a funcionalidade da pessoa portadora de deficiência" (BRASIL, 2004a, p.15).

Em 16 de novembro de 2006 foi constituído pela portaria $n^{\circ} 142$ o Comitê de Ajudas Técnicas (CAT), que é um comitê permanente criado no âmbito da Secretaria Especial dos Direitos Humanos da Presidência da República (SEDH/PR), ligado a Coordenadoria Nacional para a Integração da Pessoa Portadora de Deficiência (CORDE).

A utilização oficial do termo TA, a expressão é utilizada no singular por se tratar de uma área do conhecimento, ela foi aprovada em detrimento do CAT que entre outras ações elaborou o atual conceito de TA como sendo uma área de conhecimento interdisciplinar que engloba diferentes métodos, recursos, estratégias, produtos, praticas e serviços que têm como função principal desenvolver a funcionalidade do indivíduo (com deficiências, incapacidades ou mobilidade reduzida) tendo como objetivo principal promover qualidade de vida a inclusão social. (BRASIL, 2007b).

Esse conceito adotado até os dias atuais ressalta a característica interdisciplinar presente na TA, ou seja, não é uma área de domínio dos profissionais de saúde, como antigamente, mas sim uma área que permite o envolvimento de diferentes profissionais. Alguns autores ressaltam ainda a indefinição sobre o conceito que por ser muito ampla pode gerar confusão por invadir outros campos de conhecimento (CASTRO; SOUZA; SANTOS, 2011; GALVÃO FILHO, 2009a; GALVÃO FILHO, 2013b; BERSCH, 2013a).

Assim atualmente os serviços de TA são em sua maioria de caráter multidisciplinar e devem envolver profissionais de varias áreas como, terapeutas ocupacionais, fisioterapeutas, fonoaudiólogos, educador especial, professores e psicólogos entre outros. A parceria entre diferentes profissionais auxilia na busca da resolução de dificuldades dos alunos (BRASIL, 2007c). 


\section{Tecnologia assistiva nas escolas}

A construção da educação inclusiva necessita de uma reflexão constante por parte dos profissionais. É necessária a tomada de decisões e a criação de estratégias durante o próprio agir docente, para gerar possibilidades pedagógicas que atendam as necessidades que emergem no cotidiano da escola (CHAVIER, 2006; VITALINO; VALENTE, 2010). Nesse âmbito, a TA pode gerar alternativas e estratégias eficientes para a inclusão escolar atender e contemplar as necessidades individuais, dos alunos público-alvo da Educação Especial porque ela busca incluir o aluno ativamente em seu processo de ensino aprendizagem.

Os recursos de TA disponível na escola podem ser os recursos de acessibilidade computacional que facilitam o processamento de informações e conteúdos escolares agindo como um facilitador na aprendizagem; os recursos de comunicação alternativa que são utilizados para estudantes que apresentam comprometimentos severos de fala; auxílios de mobilidade; adequação postural, além de adaptações de recursos escolares que tenham a finalidade otimizar a funcionalidade do aluno.

Galvão Filho e Damasceno (2008), classificam os recursos de acessibilidade computacional em três elementos: adaptações físicas ou órteses, adaptações de hardware e softwares especiais de acessibilidade.

A elaboração e implementação de recursos de TA para os alunos público-alvo da Educação Especial deve seguir alguns aspectos como, por exemplo, uma avaliação preliminar, é necessário avaliar as características individuais do aluno, suas demandas motoras, orgânicas e sensoriais para posteriormente estabelecer objetivos pedagógicos que se relacionem de maneira positiva com essas características (MANIZI, DELIBERATO, 2007).

Para que as adaptações de determinados recursos surtam efeitos positivos, é necessário planejamento por parte do professor, com a participação ativa do aluno. Por isso a importância de entender as reais necessidades e capacidades do aluno. No ambiente educacional, o professor pode propor adaptações, mas sempre solicitar a opinião de seu aluno, buscando investigar as sensações e percepções do mesmo com o uso do recurso adaptado (GONÇALVES 2010).

Segundo Fachinetti, Gonçalves e Lourenço (2015) no contexto educacional a TA deve estar disponível no Atendimento Educacional Especializado e o professor pode implementar e planejar o uso de recursos de TA bem como adotar metodologia e elaborar 
estratégias de ensino condizentes com as necessidades do aluno. O Atendimento Educacional Especializado é um serviço que disponibiliza e promove condições de acesso e aprendizagem aos alunos público-alvo da Educação Especial.

Porém como a TA mostra esse caráter interdisciplinar é interessante que esse professor tenha pelo menos um profissional da saúde o auxiliando na escolha dos melhores recursos. A literatura nacional aponta sobre a importância e necessidade de programas de intervenção nas escolas, além de mostrar o quanto ações colaborativas entre diferentes profissionais como, por exemplo, os profissionais da educação e da saúde podem facilitar a inclusão dos alunos público alvo da educação especial (DELIBERATO, 2009; PELOSI, 2009 ;ROCHA, 2010).

Alguns obstáculos são apontados como responsáveis para a não utilização adequada da TA nas escolas, sendo eles a falta de financiamentos adequados, de conhecimento por parte dos profissionais bem como a falta de apoio de profissionais especializados, além de limitações em acompanhar o uso dos recursos de TA e atitudes negativas em relação aos recursos (COUPLEY; ZIVIANI, 2004; CRADDOCK, 2006).

\section{Políticas públicas que garantem a tecnologia assistiva nas escolas}

O documento Atendimento Educacional Especializado - Deficiência Física, traz material com fundamentos básicos para os professores do Atendimento Educacional Especializado, que atuam nas escolas regulares. Além de ter por objetivo oferecer orientações ao acesso a TA no cotidiano escolar de alunos com deficiência física (BRASIL, 2007c).

O Atendimento Educacional Especializado é indicado em documentos nacionais, porém alguns autores alertam que as escolas nem sempre tem um programa sistematizado envolvendo uma equipe multidisciplinar bem como os recursos de TA e consequentemente sua manutenção (DELIBERATO, 2009; PELOSI, 2009; BRACCIALLI, 2009).

O projeto de lei $n^{\circ}$ 8.035-B de 2010 aprova o Plano Nacional de educação (PNE) que tem como uma de suas metas universalizar o acesso ao atendimento escolar para toda a população, dentre as estratégias expostas para o comprimento desta meta está a garantia da disponibilização de recursos de TA.

O Programa Implantação de Sala de Recursos Multifuncionais (SRMs) (BRASIL, 2012d), por meio do Ministério da Educação e Cultura (MEC), disponibilizou para as

RPGE- Revista on line de Política e Gestão Educacional, Araraquara, v.21, n.esp.3, p. 1588-1597, dez., 2017. 
escolas públicas do ensino regular recursos de TA, visando favorecer a escolarização de estudantes público-alvo da Educação Especial, sendo esses estudantes alunos com deficiências, transtornos globais do desenvolvimento, altas habilidades/superdotação (BRASIL,2008e). De acordo com o programa de implementação das SRMs, os recursos de TA são disponibilizados nessas salas para que o professor do atendimento educacional especializado utilize com os alunos atendidos.

Recentemente foi instituída a Lei n ${ }^{\circ} 13.146$ de 06 de julho de 2015 a Lei Brasileira de Inclusão da Pessoa com Deficiência de acordo com essa nova norma jurídica estabelecida a TA é referida como: produtos, recursos, metodologias, praticas, serviços, equipamentos, dispositivos e estratégias que tenha objetivo de promover funcionalidade no indivíduo com deficiência ou mobilidade reduzida (BRASIL, 2015f). Em vários artigos do documento é garantida a utilização da TA por meio de acesso a serviços de saúde e educação em todos os níveis, bem como a inclusão no mercado de trabalho, a qualquer evento de natureza científico- cultural, a participação em processos seletivos e ao acesso a justiça do ministério público.

\section{Considerações finais}

O uso da TA bem como alterações nos matérias escolares por meio dela, pode proporcionar ao aluno público-alvo da Educação Especial condições de manuseio de objetos escolares favorável a sua necessidade, facilitando assim melhores condições de ensino aprendizagem permitindo que o aluno desempenhe atividades acadêmicas com o maior grau de funcionalidade possível.

Com relação aos recursos de TA disponibilizados nas SRMs, para auxiliar na educação dos alunos público-alvo da Educação Especial, alguns autores destacam que quando esses recursos estão presentes na SRMs, os professores relatam dificuldades em utilizar tais recursos (BERSCH, 2009b; DOUNIS, 2013; HUMMEL, 2012; LOURENÇO, 2012; MANZINI, 2011a; MANZINI, 2012b).

Outro fator que dificulta a utilização desses recursos é a burocracia administrativa, alguns recursos ficam a espera de um profissional técnico para sua instalação, ou então alguns recursos não chegam a ser entregues as escolas (GALVÃO FILHO; MIRANDA, 2012; REIS, 2013). 
O ideal seria que houvesse um serviço permanente que oferecesse diferentes profissionais capacitados para atuar diretamente com o uso da TA no ambiente educacional , afim de favorecer o processo de ensino aprendizagem dos alunos público alvo da educação especial e também garantir o caráter interdisciplinar presente no conceito de TA. Alguns autores têm discutido a importância da consultoria colaborativa como um fator essencial para a inclusão.

Segundo Mendes; Almeida e Toyoda (2011) o serviço de consultoria colaborativa tem como objetivo resolver problemas educacionais no processo de inclusão escolar e parte da relação de igualdade entre profissionais especializados e os professores que juntos podem oportunizar a construção de diferentes habilidades tanto na sua formação profissional quanto na formação do aluno, esse modelo de um serviço permanente proposto neste estudo poderia começar com base em um serviço de consultoria.

Assim concluindo esse estudo teórico, percebe-se que questões sobre formação não são o único empecilho para que se efetivem ações que favoreçam o uso da TA como facilitadora do processo de inclusão educacional. É imprescindível a necessidade de uma conceituação específica sobre a TA para que se direcione melhor as opções de serviço e principalmente financiamentos, com uma melhor conceituação das ações das políticas públicas que, como percebe-se são confusas, a utilização da TA na escola pode ser direcionada de forma mais pontual e efetiva na escolarização dos alunos público-alvo da Educação Especial.

AGRADECIMENTOS: ao CNPQ.

\section{REFERÊNCIAS}

BERSCH, R. C. R. Introdução à Tecnologia Assistiva. Porto Alegre: Assistiva Tecnologia e Educação, 2013a. Disponível em: <http://www.assistiva.com.br/Introducao_Tecnologia_Assistiva.pdf >. Acesso em: 22 jun. 2014.

BERSCH, R. C. R. Design de serviço de tecnologia assistiva em escolas públicas. 2009. 231f. Dissertação (Mestrado em Design) - Programa de Pós-Graduação em design, Universidade Federal do Rio Grande do Sul, Porto Alegre, 2009b.

BRACCIALLI, L. M. P. Intervenção precoce: contribuições da fisioterapia para área de comunicação alternativa. In: DELIBERATO, D.; GONÇALVES, M. J.; COUTINHO, E. M. (Org.). Comunicação alternativa: teoria, prática, tecnologias e pesquisa. São Paulo: Memnon, 2009, v. 1, p. 285-292. 
BRASIL. Decreto no 5.296, de 2 de dezembro de 2004. Brasília, DF: Diário Oficial da União da República Federativa do Brasil, 2004a. Disponível em:<http://www.planalto.gov.br/ccivil_03/_ato2004-2006/2004/decreto/d5296.htm>. Acesso em: 22 out. 2016.

BRASIL. Ata VII - Comitê de Ajudas Técnicas - CAT. Secretaria Especial dos Direitos Humanos da Presidência da República (CORDE/SEDH/PR). 2007b. Disponível para download em: $<$ https://docs.google.com/viewer?a=v\&pid=sites\&srcid=ZGVmYXVsdGRvbWFpbnx0 ZWNub2xvZ2lhYXNzaXN0aXZhY29tYnJ8Z3g6MTdiZWQyY2IzYTE3OWJmZg>. Acesso em: 22 ago. 2013.

BRASIL. Atendimento Educacional Especializado - Deficiência Física. Ministério da Educação. Secretaria de Educação a Distância. Secretaria de Educação Especial. Brasília: MEC, 2007c.

BRASIL. Política Nacional de Educação Especial na perspectiva da Educação Inclusiva. Ministério da Educação. Secretaria de Educação Especial. Brasília: MEC, 2008e.

BRASIL. Plano Nacional de Educação - Projeto de lei no 8.035-B de 2010. Disponível em: http://portal.mec.gov.br/index.php?option=com_content\&view=article\&id=12907:legislac oes\&catid=70\&Itemid=265:legislacoes $>$. Acesso em 14 de setembro 2016.

BRASIL. Documento Orientador Programa de Implantação de Salas de Recursos Multifuncionais. Brasília: MEC, SECADI, Diretoria de Políticas de Educação Especial, 2012d.

BRASIL. Lei no 13.146, de 06/07/2015. Institui a Lei Brasileira de Inclusão da Pessoa com Deficiência (Estatuto da pessoa com Deficiência). 2015f. Disponível em:<http://www.planalto.gov.br/ccivil_03/_Ato2015-2018/2015/Lei/L13146.htm> Acesso em: 19 agos. 2015.

CASTRO, A. S. A.; SOUZA, L. R.; SANTOS, M. C. Proposições Teóricas para a Inclusão da Tecnologia Assistiva no Currículo Escolar da Educação Básica. Sitientibus, Feira de Santana, n. 44, p. 145-158, 2011.

COPLEY, J.; ZIVIANI, J. Barriers to the use of assistive technology for children with multiple disabilities. Occupational Therapy International. United States, v.11, n.4, p. 229- 243, 2004.

CRADDOCK, G. The AT continuum in education: Novice to power user. Disability and Rehabilitation: Assistive Technology. United States, v1, n 2, p. 17-27, 2006.

DELIBERATO, D. Uso de expressões orais durante a implementação do recurso de comunicação suplementar e alternativa. Revista Brasileira de Educação Especial, v.15, p.369-388, 2009. 
DOUNIS, A. B. Atividade docente e inclusão: as mediações produzidas pela consultoria colaborativa. 2013. 180f. Dissertação (Mestrado em Educação), Universidade Federal de Alagoas, Maceió, 2013.

FACHINETTI, T.A.; GONÇALVES, A.G.; LOURENÇO, G. G. Atendimento Educacional Especializado (AEE) e a oferta para alunos com deficiência física. Crítica Educativa Sorocaba/SP, Vol.1, n.2, p. 172-186, Jul./dez. 2015.

GALVÃO FILHO, T. A.; DAMASCENO, L. L. Tecnologia assistiva em ambientecomputacional: recursos para a autonomia e inclusão sócio-digital da pessoa com deficiência.In: INSTITUTO DE TECNOLOGIA SOCIAL. Tecnologia assistiva nas escolas: recursos básicos de acessibilidade sóciodigital para pessoas com deficiência. São Paulo: Instituto de Tecnologia Social (ITS Brasil), Microsoft / Educação, 2008. p. 25-45.

GALVÃO FILHO, T. A.; MIRANDA, T. G. Tecnologia Assistiva e salas de recursos: análise crítica de um modelo. In: GALVÃO FILHO, T. A.; MIRANDA, T. G. (Orgs.). O professor e a educação inclusiva: formação, práticas e lugares. Salvador: EDUFBA, 2012, p. 247-266.

GALVÃO FILHO, T. A. Tecnologia assistiva para uma escola inclusiva: Apropriação, Demandas e Perspectivas. 2009. 346f. Tese (Doutorado em Educação), Faculdade de Educação, Universidade Federal da Bahia, Salvador, 2009a.

GALVÃO FILHO, T. A. A construção do conceito de Tecnologia Assistiva: alguns novos interrogantes e desafios. Revista Entre ideias, Salvador, v. 2, n.1, p. 25-42, 2013b.

GONÇALVES, A. G. Desempenho motor de alunos com paralisia cerebral frente à adaptação de recursos pedagógicos. 2010. 168p. Tese (Doutorado em Educação) Faculdade de Filosofia e Ciências, Unesp de Marília.

HUMMEL, E. I. Formação de professores das salas de recursos multifuncionais para o uso da tecnologia assistiva. 2012. 231f. Tese (Doutorado em Educação), Faculdade de Filosofia e Ciências, UNESP, Marília, 2012.

LOURENÇO, G. F. Avaliação de um programa de formação sobre recursos de altatecnologia assistiva e escolarização. 2012. 258f. Tese (Doutorado em Educação Especial), Centro de Ciências Humanas, Universidade Federal de São Carlos, 2012.

MANZINI, E.J.; DELIBERATO, D. Portal de ajudas técnicas: equipamento e material pedagógico especial para educação, capacitação e recreação da pessoa com deficiência física - recursos pedagógicos adaptados II. vol 1, 1. ed. Brasília: ABPEE/MEC/SEESP, 2007.

MANZINI, E. J. Formação de Professores e Tecnologia Assistiva. In: CAIADO, K. R. M.; JESUS, D. M.; BAPTISTA, C. R. (Orgs.). Professores e educação especial: formação em foco. Porto Alegre: Mediação, 2011a, v. 2, p. 45-63. 
MANZINI, E. J. Formação do professor para trabalhar com recursos de tecnologia assistiva: um estudo de caso em Mato Grosso. Educação e Fronteiras On-Line, Dourados, v.2, n.5, p.98-113, 2012b.

MENDES, E. G.; ALMEIDA, M. A.; TOYODA, C. Y. Inclusão escolar pela via da colaboração entre educação especial e educação regular. Educar em Revista (Impresso), Curitiba, v. 41, p. 80-93, 2011.

PELOSI, M. B. Tecnologias em comunicação alternativa sob o enfoque da terapia ocupacional. In: DELIBERATO, D; GONÇALVES, M. J; MACEDO, E. C. (Org.). Comunicação alternativa: teoria, prática, tecnologias e pesquisa. São Paulo: Memnon Edições Científicas, 2009. p. 163-173.

REIS, C. V. O uso de tecnologia assistiva em salas de recursos multifuncionais no sudeste goiano. In: I SIMPÓSIO INTERNACIONAL DE ESTUDOS SOBRE A DEFICIÊNCIA, 1., 2013, São Paulo. Anais. São Paulo: USP, 2013. 1 CD-ROM.

ROCHA, A. N. D. C. Processo de prescrição e confecção de recursos de tecnologia assistiva na educação infantil. 2010. 199 f. Dissertação (Mestrado em Educação) Faculdade de Filosofia e Ciências, Universidade Estadual Paulista, Marília, 2010.

RODRIGUES, P. R.; ALVES, L. R. G. Tecnologia Assistiva: uma revisão do tema. Holos, Natal, v. 6, n.29, p. 170-180, 2013.

VARELA, R. C. B.; OLIVER, F. C. A utilização de Tecnologia Assistiva na vida cotidiana de crianças com deficiência. Ciênc. saúde coletiva. Rio de Janeiro. V 18 n.6, jun 2013. Disponível em: <http://www.scielo.br/scielo. php?script=sci_arttext\&pid=S1413$81232013000600028 \& \operatorname{lng}=$ pt\&nrm=iso $>$. Acesso em: 18 set. 2017.

\section{Como referenciar este artigo}

FACHINETTI, Tamiris Aparecida.; UREL, Relma Carbone Carneiro. A Tecnologia Assistiva como facilitadora no processo de inclusão: Das Políticas Públicas a Literatura. Revista on line de Política e Gestão Educacional, Araraquara, v.21, n.esp.3, p. 15881597, dez., 2017. ISSN: 1519-9029.

Submetido em: 09/05/2017

Aprovado em: 11/07/2017 